\title{
Unravelling the molecular architecture of the Commander assembly
}

\author{
M. D. Healy ${ }^{1}$, J. Sacharz ${ }^{2}$, T. Croll ${ }^{3}$, D. A. Stroud ${ }^{2}$, B. M. Collins ${ }^{1}$ \\ ${ }^{1}$ Insitute of Molecular Biosciences, The University of QLD, Brisbane, Australia, \\ ${ }^{2}$ Department of Biochemistry and Pharmacology, University of Melbourne, Melbourne, Australia, \\ ${ }^{3}$ Cambridge Institute for Medical Research, Cambridge, United Kingdom.
}

b.collins@imb.uq.edu.au

The Commander complex is a conserved regulator of intracellular trafficking. This ancient complex consists of 15 core components as well as a number of associated proteins that can be sub-divided into 3 categories: the $C O M M D / C o i l e d$ coil domain containing protein (CCDC) 22/CCDC93 (CCC) complex, the Retriever complex (a distant relative of the Retromer complex) and a number of associated proteins. Functionally Commander couples to Sorting Nexin 17 (SNX17) to facilitate the recycling of over 100 cell surface proteins including key receptors such as, LDLR, LRP1, p-Selectin and the amyloid precursor protein. In addition, Commander dysfunction has been linked to various disease pathologies including X-linked intellectual disability. It is therefore crucial to understand the structure, mechanism and function of this complex, an understanding that has remain largely elusive to date.

In the work presented here I have followed on from our previously published work on the structure of individual COMMD proteins [1] by reconstituting the core COMMD protein complex in vitro. The successful reconstitution of this complex using a polycistronic E. coli vector has allowed for the identification of two distinct subcomplexes of the COMMD family, a result supported by in vivo experiments conducted using quantitative mass spectrometry and a panel of COMMD knockout eHAP cell lines.

Identification of these distinct subcomplexes also allowed for the resolution of a $\sim 3.3 \AA$ crystal structure of COMMD subcomplex B. Revealing an intimately assembled tetramer, with interfaces along the $\beta$-strands of the highly conserved COMM domain and more subunit specific interactions between the loops on the COMM domain and the more variable helical N-terminal domain (See Figure 1). Intriguingly despite the formation of distinct subcomplexes in in vitro expression there is sufficient evidence to suggest COMMDs exist as a decameric assembly in the endogenous cellular environment. With this in mind we have used the aforementioned structure to model this assembly, revealing a geometrically perfect star assembly (See Figure 2).

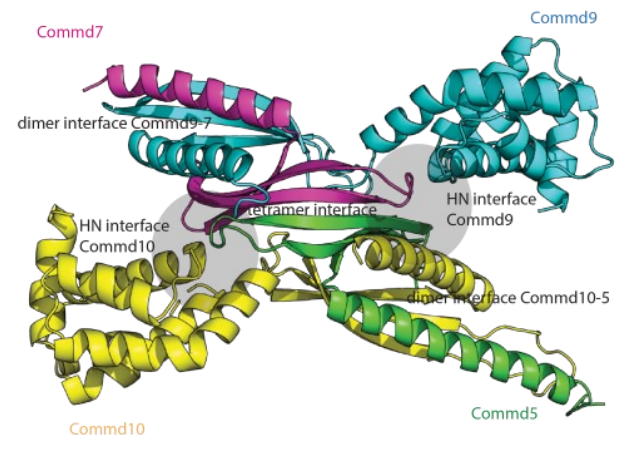

Figure 1. 3.3 $\AA$ structure of COMMD subcomplex B, reveals a tightly assembled tetramer with conserved and subunit specific interfaces.

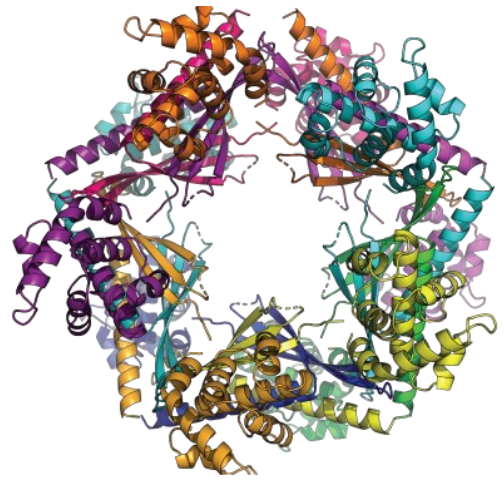

Figure 2. Model of the COMMD protein decameric assembly.

[1] Healy, M. D., Hospenthal, M. K., Hall, R. J., Chandra, M., Chilton, M., Tillu, V., Chen, K., Celligoi, D. J., McDonald, F. J., Cullen, P. J., Lott, J. S., Collins, B. M., Ghai, R. (2018). eLIFE. 7, e35895.

Keywords: Crystallography; Membrane Trafficking; Multidisciplinary 